\title{
The chemometrics approach applied to FTIR spectral data for the analysis of rice bran oil in extra virgin olive oil
}

\begin{abstract}
Among eleven studied vegetable oils, rice bran oil (RBO) has the close similarity to extra virgin olive oil (EVOO) in terms of FTIR spectra, as shown in the score plot of first and second principal components. The peak intensities at 18 frequency regions were used as matrix variables in principal component analysis (PCA). Consequently, the presence of RBO in EVOO is difficult to detect. This study aimed to use the chemometrics approach, namely discriminant analysis (DA) and multivariate calibrations of partial least square and principle component regression to analyze RBO in EVOO. DA was used for the classification of EVOO and EVOO mixed with RBO. Multivariate calibrations were exploited for the quantification of RBO in EVOO. The combined frequency regions of 1200-900 and 3020$3000 \mathrm{~cm}-1$ were used for such analysis. The results showed that no misclassification was reported for the classification of EVOO and EVOO mixed with RBO. Partial least square regression either using normal or first derivative FTIR spectra can be successfully used for the quantification of RBO in EVOO. In addition, analysis of fatty acid composition can complement the results obtained from FTIR spectral data.
\end{abstract}

Keyword: Chemometrics; FTIR spectroscopy; Rice bran oil; Extra virgin olive oil 